'Servicio de Neurología, Hospital Clínico Regional de Concepción "Dr. Guillermo Grant Benavente". Concepción, Chile. ${ }^{2}$ Departamento de Especialidades, Facultad de Medicina, Universidad de Concepción. Concepción, Chile. ${ }^{3}$ Departamento de Salud Pública, Facultad de Medicina, Universidad de Concepción. Concepción, Chile. ${ }^{4}$ Departamento de Educación Médica, Facultad de Medicina, Universidad de Concepción. Concepción, Chile. apsicólogo. ${ }^{\text {bMatrón. }}$ cPhD.

${ }^{d}$ Candidato a Doctor en Epidemiología y Salud Pública por la Universidad Autónoma de Madrid, España.

Financiamiento: Este estudio se enmarca dentro del proyecto VRID 218.083.034-1.02017 financiado por la Vicerrectoría de Investigación y Desarrollo de la Universidad de Concepción, Chile. No existen conflictos de interés con la fuente de financiamiento de esta investigación.

Recibido el 26 de junio de 2019, aceptado el 7 de mayo de 2020

Correspondencia a: Mario Fuentealba Sandoval Departamento de Especialidades. Facultad de Medicina. Universidad de Concepción. Avda. Chacabuco esquina Janequeo $s / n, 4^{\circ}$ piso. Concepción, Chile. mariovfu@udec.cl

\section{Factores asociados a respuesta favorable de tratamiento corticoidal en pacientes con polirradiculoneuropatía desmielinizante inflamatoria crónica}

\author{
MARIO FUENTEALBA S. ${ }^{1,2}$, IGNACIO MOLINA D. ${ }^{1}$, \\ ALDO VERA-CALZARETTA ${ }^{3, a, c}$, CRISTHIAN PÉREZ-VILLALOBOS $^{4, a, c}$, \\ GONZALO KLAASSEN P. ${ }^{1,2}$, SERGIO JUICA A. ${ }^{1,2}$, \\ ALEXIS GONZÁLEZ-BURBOA ${ }^{3, \mathrm{~b}, \mathrm{~d}}$
}

\section{Variables associated with a good response to steroids in chronic inflammatory demyelinating polyradiculoneuropathy}

Background: The treatment of chronic inflammatory demyelinating polyradiculoneuropathy (CIDP) is based on corticosteroids, immunoglobulin and plasmapheresis. In our Health System, corticosteroids are commonly used as first line therapy for economic reasons and accessibility. However, the factors associated with a good response are not well known. Aim: To assess the association of demographic, clinical and laboratory variables with a favorable response to corticosteroid therapy in patients with CIDP. Material and Methods: Observational, descriptive, longitudinal and retrospective study of 33 patients with a diagnosis of typical, definitive or probable CIDP, treated with corticosteroids for at least six months. Results: Twenty-three patients had a good clinical response to corticosteroid treatment and 10 were non-responders. The variables significantly associated with a good response to steroids were a disease lasting less than 1 year prior to the start of treatment, the absence of axonal damage in electromyography a relapsing-recurrent course and a favorable response within two months of treatment. Conclusions: Most of these patients with CIDP had good response to corticosteroid therapy.

(Rev Med Chile 2020; 148: 594-601)

Key words: Corticosteroids; Polyradiculoneuropathy, Chronic Inflammatory Demyelinating; Therapeutics.
$\mathrm{L}$ a polirradiculoneuropatía desmielinizante inflamatoria crónica (CIDP) es la neuropatía autoinmune periférica más frecuente, con una prevalencia variable $(0,7$ a 10,3 por cada 100.000 habitantes con un promedio agrupado de $2,87(1,58-4,39)$ siendo en gran parte esta variabilidad debida a los diferentes criterios clínicos recomendados para su diagnóstico ${ }^{1,2}$.

EL CIDP es una polineuropatía crónica pro- gresiva, recurrente-remitente y escalonada, a menudo incapacitante y más de $50 \%$ de los pacientes presentan discapacidad temporal y alrededor del $10 \%$ discapacidad permanente ${ }^{3}$.

Los corticosteroides, la plasmaféresis, las inmunoglobulinas intravenosas y ahora subcutáneas son tratamientos eficaces comprobados para la CIDP.

La probabilidad de mejoría después de estos 
tratamientos varía entre $50 \%$ a $80 \%$ aproximadamente ${ }^{5-7}$ un reciente estudio demostró la utilidad de la inmunoglobulina subcutánea ${ }^{8}$ ensayos clínicos aleatorios no han demostrado que otros fármacos inmunosupresores sean efectivos en el tratamiento de la CIDP, como el metotrexato, la azatioprina y la ciclosporina'.

Si bien se considera que los efectos secundarios de la inmunoglobulina intravenosa son generalmente menores y, potencialmente, más frecuentes y graves después del uso a largo plazo de los corticosteroides, distintos ensayos prospectivos y aleatorizados muestran que los efectos adversos del tratamiento corticoidal a corto plazo son menores y transitorios, resolviéndose espontáneamente después de suspender la terapia con corticoides ${ }^{10}$.

El primer reporte de un caso de polineuropatía recurrente respondedora a corticoides es el trabajo seminal en este tema ${ }^{11}$ siendo el tratamiento esteroidal el más usado a nivel mundial, fundamentalmente por la accesibilidad y la notable diferencia económica entre los terapias disponibles ${ }^{12}$. Práctica que se ha fortalecido con datos consistentes que muestran que los corticosteroides tienen un mayor potencial para lograr una remisión clínica que permita suspender la terapia, o períodos de remisión más largos comparados con la inmunoglobulina ${ }^{13}$.

Existe poca evidencia sobre los predictores de la respuesta a los corticosteroides en pacientes con $\mathrm{CIDP}^{14-18}$. Las series de pacientes son heterogéneas en cuanto a los participantes incluidos y los factores que predijeron una mejor respuesta a la terapia CIDP con tratamiento corticoidal.

Si bien se han confeccionado algoritmos terapéuticos para el manejo del CIDP en algunos países, la controversia persiste hasta el día de hoy $^{19,20}$, no existiendo evidencia de calidad con respecto a la elección del tratamiento de primera línea del CIDP.

Este estudio tiene como objetivo caracterizar una cohorte de pacientes con diagnóstico de CIDP según criterios cínicos por EFNS-EPS ${ }^{21}$, de la base datos del policlínico neuromuscular del HGGB, Dividir dicha cohorte entre respondedores y no respondedores a tratamiento corticoidal, durante aplicando criterios estandarizados para evaluar el resultado terapéutico y establecer la relación entre antecedentes mórbidos, manifestaciones clínicas, y exámenes de laboratorio, respecto a la remisión clínica de enfermedad.

\section{Material y Método}

\section{Tipo de estudio}

Observacional, descriptivo, longitudinal, retrospectivo de serie de casos.

\section{Clasificación \\ Investigación cuantitativa.}

\section{Población y muestra:}

Pacientes mayores de 18 años con diagnóstico de CIDP del HGGB, desde 2001 a 2017, que hayan sido sometidos a terapia corticoidal por un mínimo de 6 meses, y que cumplan con los criterios de inclusión. Del universo total de 43 pacientes registrados en el policlínico de neuromuscular del HGGB con diagnóstico de egreso DE CIDP, 8 habían fallecido de los 35 restantes, 1 paciente no fue tratado con terapia corticoidal y 1 paciente se negó a participar, por lo que la población final a fue de 33 pacientes.

\section{Criterios de inclusión y exclusión}

\section{Criterios de inclusión}

- Paciente con diagnóstico de CIDP según categorías diagnósticas (Tabla 6) EFNS-EPS ${ }^{21}$ clínicos típico (típico (definido y probable).

- Mayor de 18 años.

- Seguimiento y tratamiento corticoidal por al menos 6 meses.

\section{Criterios de exclusión}

- Indicación de terapia corticoidal por otra enfermedad autoinmune.

- Negativa a firmar consentimiento informado de participación en el estudio.

- Fallecimiento previo a la evaluación clínica enmarcada en este estudio.

\section{Criterios de paciente respondedor y no respondedor}

\section{Criterios de paciente respondedor}

Al momento de la evaluación, mejoría de al menos 2 puntos en cualquier músculo en la escala de la Medical Research Council (MRC) ${ }^{22}$ y/o de al menos 1 punto en la escala de Hughes ${ }^{23}$, y/o de dos puntos en la escala de discapacidad ajustada de causa y tratamiento de la neuropatía inflamatoria $(\text { INCAT })^{24}$, respecto a evaluación registrada previa al inicio del tratamiento. 


\section{Criterios de paciente No respondedor}

$\mathrm{Al}$ momento de la evaluación, paciente mantiene o empeora puntaje de escalas estandarizadas (MRC, Hughes, INCAT), respecto a evaluación registrada previa al inicio del tratamiento.

\section{Criterios de lesión axonal en electromiografía cercana a la evaluación ${ }^{25}$}

Presencia de abundantes potenciales polifásicos de gran amplitud y duración con patrón de reclutamiento mixto o empobrecido presente en $2 \mathrm{o}+$ nervios en más de 1 extremidad.

\section{Consideraciones éticas}

Fue aprobado por el Comité Ético Científico del Servicio de Salud Concepción (Resolución exenta No 848, No 609, Código 18-11-88), se elaboró una ficha informativa sobre los objetivos generales y se aplicó consentimiento informado a la totalidad de pacientes, se diseñó un formulario de recolección de datos asignando un número de folio a cada sujeto, resguardando la calidad de confidencialidad de la información proporcionada.

Instrumento de recogida de Información: Previa coordinación con el investigador, se citó a los sujetos de estudio, se recabaron datos sociodemográficos y clínicos, se aplicaron escalas estandarizadas (evaluación) se revisaron fichas médicas del archivo del hospital y de ficha electrónica.

\section{Plan de análisis}

Base de datos en Excel con la información recogida de las 24 variables definidas para caracterizar la cohorte, siendo la respuesta clínica de enfermedad la variable de interés, para ser exportada a la suite estadística SPSS versión 21. Se realizó análisis exploratorio de los datos, utilizando porcentajes, distribución, media aritmética, y rangos. Se utilizaron pruebas no paramétricas para establecer una correlación bivariada entre la remisión clínica de enfermedad y 20 de las variables a estudiar, con un intervalo de confianza de $95 \%$. En caso de variables cualitativas, se utilizó la prueba exacta de Fisher. Al momento de analizar la correlación entre la remisión clínica y variables cuantitativas, se utilizó la prueba de la U de Mann-Whitney.

\section{Resultados}

Veintitrés casos cumplieron con los criterios de paciente respondedor, siendo 10 los no res- pondedores. El promedio al inicio del tratamiento fue de 52,3 \pm 18 años. 19 pacientes eran de sexo masculino entre las comorbilidades más frecuentes se identificaron la hipertensión arterial $(n=12)$, diabetes $(n=9)$, neoplasia $(n=8)$, dislipidemia $(\mathrm{n}=6)$, hipotiroidismo $(\mathrm{n}=5)$, artrosis $(\mathrm{n}=4)$, arritmia $(\mathrm{n}=3)$ y trastorno del ánimo $(\mathrm{n}=2)$.

Según los datos analizados, los antecedentes demográficos y clínicos como la edad, el sexo, la presencia de diabetes no tendrían relación con la respuesta a terapia corticoidal $(\mathrm{p}>0,05$; variables independientes entre sí) (Tabla 1 ).

Respecto a las características clínicas del episodio inicial, 27 pacientes presentaron un inicio crónico definido como una evolución mayor a dos meses, 2 pacientes presentaron inicio subagudo (mayor deterioro clínico entre el primer y segundo mes) y 4 debutaron con un episodio inicial agudo (evolución menor a un mes). La distribución del compromiso neurológico fue simétrica en 21 casos y relativamente asimétrica en 12. La presencia de compromiso sensitivo se identificó en 19 pacientes. En 7 pacientes hubo alteración de pares craneanos. En 22 casos la región más afectada fue distal, en 1 caso fue proximal y en 10 el componente fue mixto. 15 pacientes presentaron un tiempo de evolución mayor a 1 año previo al inicio del tratamiento, mientras que 18 pacientes fueron tratados antes de cumplir 12 meses desde el inicio de la sintomatología. Al analizar estas variables sólo se observó una relación significativa entre el grupo que recibió tratamiento antes de 1 año de iniciada la patología y la remisión clínica (Tabla 2).

Se identificó disociación albúmino-citológica en el líquido cefalorraquídeo (proteinorraquia mayor de $0,45 \mathrm{~g} / \mathrm{L}$, con celularidad no mayor a 10 leucocitos $/ \mathrm{mm}^{3}$ ) en 26 pacientes no encontrándose relacionada con la remisión clínica. Se detectó daño axonal en estudio electromiográfico en 17 pacientes observándose una relación significativa respecto a la remisión clínica (Tabla 3 ).

Se administró esquema de prednisona $1 \mathrm{mg} /$ $\mathrm{kg}$ diario por 2 meses en todos los pacientes, pasando a régimen alterno entre los 2 y los 6 meses en 24 pacientes y en 25 se agrega como terapia de mantención inmunosupresores (la gran mayoría azatioprina) en los casos de inicio subagudo se administra metilprednisolona $1 \mathrm{~g}$ por 3 días como inducción seguida del régimen oral. Doce pacientes recibieron inmunoglobulina endovenosa en 
Tabla 1. Antecedentes demográficos y clínicos según respuesta terapéutica $(n=33)$

\begin{tabular}{|c|c|c|c|c|}
\hline & Total & Respondedor (23) & No respondedor $(10)$ & Valor $\mathbf{p}$ \\
\hline $\begin{array}{l}\text { Edad al inicio de terapia } \\
\qquad \text { Años } \pm D E^{1}\end{array}$ & $52,3 \pm 18$ & $49,1 \pm 18$ & $59,5 \pm 16,8$ & $0,142^{*}$ \\
\hline $\begin{array}{l}\text { Sexo, n (\%) } \\
\text { Masculino }\end{array}$ & $19(58)$ & $12(53)$ & $7(70)$ & $0,287 * *$ \\
\hline $\begin{array}{l}\text { Diabetes, n (\%) } \\
\text { Presente }\end{array}$ & $9(27)$ & $5(22)$ & $4(40)$ & $0,4^{* *}$ \\
\hline
\end{tabular}

1Desviación estándar; *Prueba U de Mann-Whitney; **Test exacto de Fisher.

Tabla 2. Características clínicas en relación a respuesta a terapia $(n=33)$

\begin{tabular}{|c|c|c|c|c|}
\hline Presentación clínica & Total & Respondedor & No respondedor & Valor $\mathbf{p}$ \\
\hline \multicolumn{5}{|c|}{ Cuadro clínico inicial, n (\%) } \\
\hline Crónico & $27(82)$ & $19(83)$ & $8(80)$ & $1,0^{*}$ \\
\hline \multicolumn{5}{|l|}{ Distribución, n (\%) } \\
\hline Simétrica & $21(64)$ & $17(74)$ & $4(40)$ & $0,114^{*}$ \\
\hline \multicolumn{5}{|c|}{ Compromiso sensitivo, n (\%) } \\
\hline Presente & $19(58)$ & $14(61)$ & $5(50)$ & $0,707^{*}$ \\
\hline \multicolumn{5}{|c|}{ Compromiso de pares craneanos, n (\%) } \\
\hline Presente & $7(21)$ & $6(26)$ & $1(10)$ & $0,397 *$ \\
\hline \multicolumn{5}{|l|}{ Región afectada, n (\%) } \\
\hline Distal & $22(67)$ & $14(61)$ & $8(80)$ & $0,430 *$ \\
\hline \multicolumn{5}{|c|}{ Tiempo de evolución previo al inicio del tratamiento, n (\%) } \\
\hline Mayor a 1 año & $15(46)$ & $7(30)$ & $8(80)$ & $0,020^{*}$ \\
\hline \multicolumn{5}{|l|}{ Curso evolutivo, n (\%) } \\
\hline Progresivo & $4(12)$ & $0(0)$ & $4(40)$ & $0,005^{*}$ \\
\hline
\end{tabular}

*Test exacto de Fisher.

Tabla 3. Resultado de exámenes en relación a remisión clínica $(\mathbf{n}=\mathbf{3 3})$

\begin{tabular}{|lcccc|}
\hline & Total & Respondedor & No respondedor & Valor $\mathbf{p}$ \\
$\begin{array}{l}\text { Disociación albúmino-citológica }{ }^{1}, \mathrm{n}(\%) \\
\text { Presente }\end{array}$ & $26(79)$ & $19(83)$ & $7(70)$ & $0,64^{*}$ \\
$\begin{array}{l}\text { Daño axonal en } \mathrm{EMG}^{2}, \mathrm{n}(\%) \\
\quad \text { Presente }\end{array}$ & $17(52)$ & $9(39)$ & $8(80)$ & $0,05^{*}$ \\
\hline
\end{tabular}

'Valor > 0,45; ${ }^{2}$ Electromiografía; *Test exacto de Fisher.

todos los casos después de los 2 meses o 1 mes antes del inicio de la terapia.

En todos los casos se completó un mínimo de 6 meses de corticoides. Del total de pacientes, $26(4 / 5)$ presentaron rápida respuesta inicial dentro de los 2 meses de iniciada la terapia con corticoides .
Veintidós pacientes presentaron en algún momento efectos adversos al medicamento, la mayoría leves no modificó el plan terapéutico y efectos adversos severos en 5 casos, hiperglicemia, osteoporosis, psicosis, hipercortisolismo e infección asociada al tratamiento, siendo suspendida la terapia. 
Factores asociados a respuesta favorable a corticoides en CIDP - M. Fuentealba et al

Tabla 4. Variables relacionadas a la terapia según respuesta clínica $(\mathbf{n}=33)$

\begin{tabular}{|c|c|c|c|c|}
\hline & Total & Respondedor & No respondedor & Valor $p$ \\
\hline $\begin{array}{l}\text { Buena respuesta inicial a corticoides, n (\%) } \\
\text { Presente }\end{array}$ & $26(79)$ & $21(91)$ & $5(50)$ & $0,016^{*}$ \\
\hline $\begin{array}{l}\text { Administración de inmunoglobulina n (\%) } \\
\text { Sí }\end{array}$ & $12(36)$ & $7(30)$ & $5(50)$ & $0,433^{*}$ \\
\hline $\begin{array}{l}\text { Terapia con inmunosupresores, n (\%) } \\
\text { Sí }\end{array}$ & $25(76)$ & $16(70)$ & $9(90)$ & $0,177^{*}$ \\
\hline
\end{tabular}

*Test exacto de Fisher.

Tabla 5. Aspectos de la evolución relacionadas con la remisión clínica $(\mathbf{n}=33$ )

\begin{tabular}{|lcccc|}
\hline Evolución clínica & Total & Remisión clínica & Sin remisión & Valor p \\
\hline Tiempo total transcurrido (años), $\pm \mathrm{DE}$ & $4,3 \pm 2,6$ & $4,3 \pm 2,4$ & $4,2 \pm 3,1$ & $0,620^{*}$ \\
$\begin{array}{l}\text { Curso evolutivo, } \mathrm{n}(\%) \\
\quad \text { Progresivo }\end{array}$ & $4(12,1)$ & 0 & & \\
$\quad$ Remitente-recurrente & $29(87,9)$ & $23(100)$ & $4(40)$ & $0,005^{* *}$ \\
\hline
\end{tabular}

*Prueba U de Mann-Whitney; **Test exacto de Fisher; ${ }^{*}$ Según los valores esperados para sexo y edad.

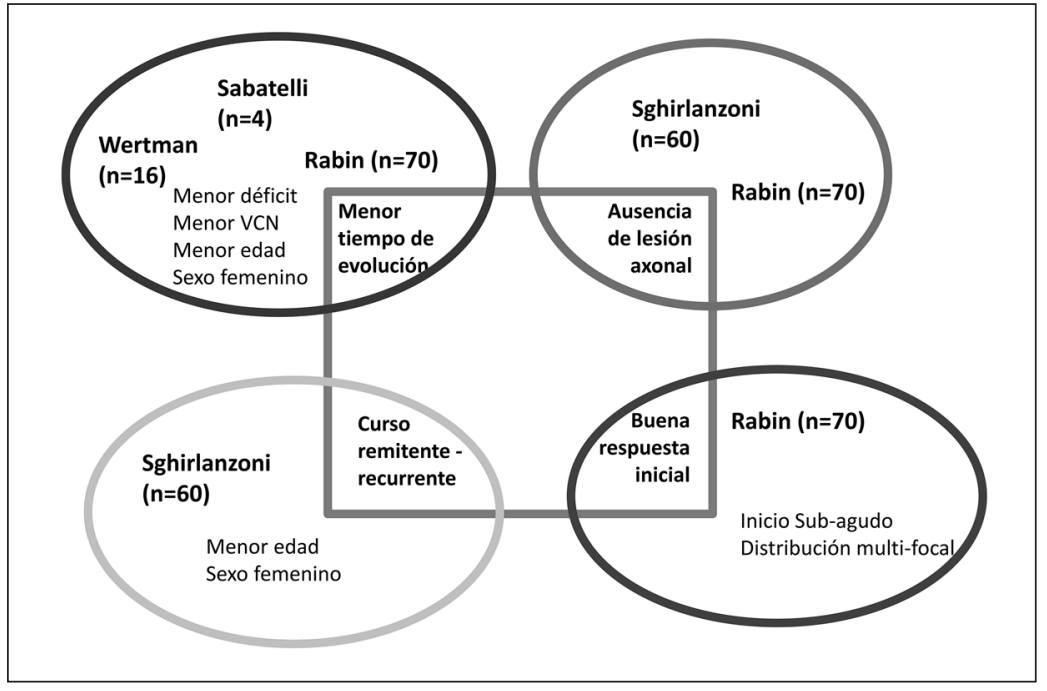

Figura 1. Comparación de trabajos predictores de respuesta corticoidal en $\mathrm{CIDP}^{13-18}$.
Respecto al tratamiento, destaca la relación observada entre una buena respuesta inicial a corticoides y la remisión clínica de enfermedad a mediano/largo plazo $(\mathrm{p}<0,016)$, no encontrándose relación con las otras variables observadas (Tabla 4).

El tiempo total transcurrido desde el inicio del cuadro hasta el momento de la evaluación enmarcada en este estudio fue de 4,3 $\pm 2,6$ años, sin presentar diferencias significativas entre pacientes respondedores y no respondedores. En cuanto al curso evolutivo, 29 pacientes presentaron un patrón remitente-recurrente, mientras que 4 casos evolucionaron de forma progresiva. Destaca la significativa relación entre el curso progresivo de la enfermedad y la mala respuesta a tratamiento corticoidal. 


\section{Discusión}

El CIDP es una patología que a lo largo de los años ha presentado un reto diagnóstico y terapéutico para los neurólogos, actualmente se cuentan con terapias eficaces como los corticoides orales y endovenosos, la plasmaféresis, la inmunoglobulina endovenosa y más recientemente la inmunoglobulina subcutánea, así como nuevas terapias en investigación como rituximab ${ }^{26}$. El tratamiento a largo plazo con corticosteroides puede tener efectos secundarios severos y la inmunoglobulina intravenosa y la inmunoglobulina subcutánea son costosas y su tolerancia no es óptima. Según un reciente estudio en que se hizo un análisis de costo utilidad de corticoides versus inmunoglobulinas, el costo incremental de las inmunoglobulinas es abrumadoramente mayor que para los corticoi$\operatorname{des}^{27}$. Si bien ambas son igualmente eficaces el costo económico hace que la terapia corticoidal sea la terapia de primera línea en CIDP en los hospitales públicos. Si se decide el tratamiento con corticosteroides, hay varios regímenes disponibles que parecen ser igualmente efectivos: prednisona, prednisolona oral, dexametasona pulsada en dosis altas o metilprednisolona intravenosa. No se ha demostrado que un tratamiento específico sea el más efectivo y parecen relativamente seguros cuando se usan por un plazo acotado e durante tiempo prolongado con metilprednisolona endovenosa que parece presentar el mejor perfil ${ }^{28,29}$.

Diversos trabajos han tratado de identificar factores asociados a la respuesta terapéutica corticoidal. Wertman ${ }^{13}$ estudió 16 pacientes, encontrando que los factores que se correlacionan con una mejor respuesta al corticoide son; la menor duración de la CIDP, menor déficit neurológico, menor disminución de la velocidad de conducción nerviosa, menor edad y el ser mujer. De las variables mencionadas en nuestro trabajo encontramos como factor asociado la menor duración del CIDP previo al inicio del tratamiento, Muley ${ }^{14}$ publicó una serie de 10 casos, sin embargo, no identificó una relación significativa entre la remisión de enfermedad y las variables que estudió, Sghirlanzoni ${ }^{15}$ publicó el estudio predictivo con mayor número de pacientes a la fecha, concluyendo que las siguientes variables fueron predictivas de un mejor resultado a la terapia: el sexo femenino, la edad más temprana al inicio, el curso remitente-recurrente y la ausencia de daño axonal en el estudio neurofisiológico. Dichos resultados coinciden con nuestro estudio, respecto al hallazgo de una relación importante entre el curso remitente-recurrente y la remisión de la enfermedad y una asociación significativa entre la ausencia de daño axonal y la buena respuesta a la terapia, sin embargo no encontramos relación respecto al sexo, ni con la edad de inicio Rajabally ${ }^{16}$ concluyó a través de un estudio en 14 pacientes que aquellos que presentaban afectación de la vía motora y preservación de sensibilidad tendrían más probabilidades de presentar una buena respuesta a la terapia, en nuestros resultados, el compromiso sensitivo no tuvo una relación significativa con la remisión de enfermedad. Sabatelli ${ }^{17}$ al contrario, describió una serie de 4 pacientes que sólo presentaban compromiso motor y ninguno de ellos respondió favorablemente a los corticoides. los 4 presentaban larga evolución de la enfermedad, variable que en nuestro estudio fue relacionada a una mala respuesta a la terapia corticoidal. Rabin ${ }^{18}$ describió en un meta-análisis que el principal factor terapéutico que influyó en el retiro del tratamiento fue el retraso en el inicio del tratamiento efectivo lo que es coincidente con nuestra serie. Además, describe que la tasa de retirada exitosa de terapia fue menor con inmunoglobulina endovenosa que con los corticosteroides, y destaca el hecho que una buena respuesta al tratamiento inicial con corticosteroides fue más frecuente en pacientes que pudieron interrumpir el tratamiento con remisión completa. Dichos resultados son similares a los obtenidos en nuestro estudio, en donde la rápida respuesta a corticoides se relacionó a una mejor respuesta. Rabin describe que un déficit asimétrico y la aparición subaguda no presentó relación significativa tras el análisis multivariado lo que coincide con lo observado en nuestro estudio.

Es una decisión costo/efectiva adecuada considerar a los corticoides como terapia de primera línea en los pacientes con CIDP, por lo que seleccionando adecuadamente nuestros pacientes se podría destinar el uso de la inmunoglobulina endovenosa a los pacientes que presenten una mala respuesta inicial al tratamiento con corticoides, o bien, sean diagnosticados tardíamente, con una evolución mayor de 1 año de la enfermedad especialmente si su curso es crónico progresivo o que tengan lesión axonal secundaria en la electromiografía asimismo considerar pacientes con riesgo por enfermedades previas o que hayan presentado 
reacciones adversas severas a los corticoides.

La principal limitación es el diseño observacional y retrospectivo de serie de casos (la misma situación de la mayoría de las otras series) con sesgos sistemáticos inherentes de este diseño. Otra posible limitación es la diferencia en el tiempo de tratamiento y seguimiento de los pacientes aunque la mayoría tuvo plan terapéutico standard. Siempre es un desafío diagnóstico el CIDP especialmente en los casos atípicos ${ }^{30}$ que fueron excluidos del estudio, pero tenemos presente que aun adscribiendo a las recomendaciones de EFNS existe la posibilidad de error diagnóstico.

Este estudio provee información de nuestra población que puede ser útil para los neurólogos que tratan pacientes con CIDP y que se ven enfrentados a las preguntas ¿cuál es la indicación de primera línea en el tratamiento del CIDP? ¿cuáles son las características de los pacientes con CIDP que responden mejor a corticoides?

Es necesario impulsar protocolos de ensayos clínicos aleatorizados (ECA) que permitan obtener evidencia de calidad e idealmente revisiones sistemáticas (RS) para construir guías clínicas de manejo validadas universalmente.

\section{Referencias}

1. MP, Manji H, Choudhary PP, Hughes RA, Thomas PK. Chronic inflammatory demyelinating polyradiculoneuropathy: a prevalence study in south east England. J Neurol Neurosurg Psychiatry 1999; 66: 677-80.

2. Broers MC, Bunschoten C, Nieboer D, Lingsma HF, Jacobs BC. Incidence and Prevalence of Chronic Inflammatory Demyelinating Polyradiculoneuropathy: A Systematic Review and Meta-Analysis. Neuroepidemiology 2019; 52 (3-4): 161-72.

3. Chiò A, Cocito D, Bottacchi E, Buffa C, Leone M, Plano $\mathrm{F}$, et al. Idiopathic chronic inflammatory demyelinating polyneuropathy: an epidemiological study in Italythe PARCIDP. J Neurol Neurosurg Psychiatry 2007; 78 (12): 1349-53.

4. Oaklander AL, Lunn MP, Hughes RA, van Schaik IN, Frost C, Chalk CH. Treatments for chronic inflammatory demyelinating polyradiculoneuropathy (CIDP): an overview of systematic reviews. Cochrane Database Syst Rev 2017; 1 (1): CD010369.

5. Eftimov F, Winer JB, Vermeulen M, de Haan R, van Schaik IN. Intravenous immunoglobulin for chronic inflammatory demyelinating polyradiculoneuropathy.
Cochrane Database Syst Rev 2013; (12): CD001797.

6. Hughes RA, Mehndiratta MM, Rajabally YA. Corticosteroids for chronic inflammatory demyelinating polyradiculoneuropathy. Cochrane Database Syst Rev 2017; 11 (11): CD002062.

7. Mehndiratta MM, Hughes RAC, Pritchard J. Plasma exchange for chronic inflammatory demyelinating polyradiculoneuropathy. Cochrane Database Syst Rev 2015; 2015 (8): CD003906.

8. van Schaik IN. Subcutaneous immunoglobulin for maintenance treatment in chronic inflammatory demyelinating polyneuropathy (PATH): a randomised, double-blind, placebo-controlled, phase 3 trial. Neurol 2018; 17 (1): 35-46.

9. Mahdi-Rogers M, Brassington R, Gunn AA, van Doorn PA, Hughes RA. Immunomodulatory treatment other than corticosteroids, immunoglobulin and plasma exchange for chronic inflammatory demyelinating polyradiculoneuropathy. Cochrane Database Syst Rev 2017; 5 (5): CD003280.

10. Press R, Hiew FL, Rajabally YA. Steroids for chronic inflammatory demyelinating polyradiculoneuropathy: evidence base and clinical practice. Acta Neurol Scand 2016: 133: 228-38.

11. Austin JH. Recurrent polyneuropathies and their corticosteroid treatment: with five-year observations of a placebo controlled case treated with corticotrophin, cortisone, and prednisone. Brain 1958; 81: 157-92.

12. McCrone P, Chisholm D, Knapp M, Hughes R, Comi G, Dalakas MC, et al. INCAT Study Group. Cost-utility analysis of intravenous immunoglobulin and prednisolone for chronic inflammatory demyelinating polyradiculoneuropathy. European Journal of Neurology 2003; 10 (6): 687-9.

13. Nobile-Orazio, Dario Cocito, Stefano Jann, Antonino Uncini, Ettore Beghi, Paolo Messina Intravenous immunoglobulin versus intravenous methylprednisolone for chronic inflammatory demyelinating polyradiculoneuropathy: a randomised controlled trial. Lancet Neurol 2012; 11: 493-502.

14. Wertman E, Argov Z, Abrmasky O. Chronic inflammatory demyelinating polyradiculoneuropathy: features and prognostic factors with corticosteroid therapy. Eur Neurol 1988; 28: 199-204.

15. Muley SA, Kelkar P, Parry GJ. Treatment of chronic inflammatory demyelinating polyneuropathy with pulsed oral steroids. Arch Neurol 2008; 65: 1460-4.

16. Sghirlanzoni A, Solari A, Ciano C, Mariotti C, Fallica E, Pareyson D. Chronic inflammatory demyelinating polyneuropathy: long-term course and treatment of 60 patients. Neurol Sci 2000; 21: 31-7. 
17. Rajabally YA, Narasimhan M, Chavada G. Electrophysiological predictors of steroid-responsiveness in chronic inflammatory demyelinating polyneuropathy. J Neurol 2008; 255: 936-8.

18. Sabatelli M, Madia M, Mignogna T, Lippi G, Quaranta 1, Tonali P. Pure motor chronic inflammatory demyelinating polyneuropathy. J Neurol 2001; 248: 772-7.

19. Rabin M, Mutlu G, Stojkovic T, Maisonobe T, Lenglet T, Fournier E, Bouche P, Léger J, Viala K. Chronic inflammatory demyelinating polyradiculoneuropathy: search for factors associated with treatment dependence or successful withdrawal. J Neurol Neurosurg Psychiatry 2013; 0: 1-6.

20. Lehmann HC, Burke D, Kuwabara S. Chronic inflammatory demyelinating polyneuropathy: update on diagnosis, immunopathogenesis and treatment. J Neurol Neurosurg Psychiatry 2019; 0: 1-7.

21. Van den Bergh PY, Hadden RD, Bouche P, Cornblath DR, Hahn A, Illa I. European Federation of Neurological Societies/Peripheral Nerve Society guideline on management of chronic inflammatory demyelinating polyradiculoneuropathy: report of a joint task force of the European Federation of Neurological Societies and the Peripheral Nerve Society: first revision. Eur J Neurol 2010; 17: 356-63.

22. Alastair Compston, Aids to the Investigation of Peripheral Nerve Injuries. Medical Research Council: Nerve Injuries Research Committee. Memorando No. 45. London, Pendragon House 1976; 6-7.

23. Hughes RAC, Cornblath DR. Guillain-Barre syndrome. Lancet. 2005; 366 (9497): 1653-66. Progress in diagnosis and treatment of chronic inflammatory demyelinating polyradiculoneuropathy.

24. Merkies IS, Schmitz PI, van der Meche FG, Samijn JP, van Doorn PA. Clinimetric evaluation of a new overall disability scale in immune mediated polyneuropathies. J Neurol Neurosurg Psychiatry 2002; 72 (5): 596-601.

25. Artieda J. Electromiografía en: Jorge Iriarte Franco, Julio Artieda González-Granda editores Manual de Neurofisiología Clínica Editorial Panamericana; 2003 pág 111 .

26. Roux, Debs R, Maisonobe T, Lenglet $\mathrm{T}$, et al. Rituximab in chronic inflammatory demyelinating polyradiculoneuropathy with associated diseases Peripher Nerv Syst. 2018; 23 (4): 235-40. doi: 10.1111/jns.12287. Epub 2018 Oct 7.

27. Blackhouse G, Gaebel K, Xie F, Campbell K, Assasi N, Tarride J-E, et al. Cost-utility of Intravenous Immunoglobulin (IVIG) compared with corticosteroids for the treatment of Chronic Inflammatory Demyelinating Polyneuropathy (CIDP) in Canada https://www.ncbi. nlm.nih.gov/pmc/articles/PMC2903512/\#

28. van Lieverloo GGA, Peric S, Doneddu PE, Gallia F, Nikolic A, Wieske L, et al. Corticosteroids in chronic inflammatory demyelinating polyneuropathy: a retrospective, multicentre study, comparing efficacy and safety of daily prednisolone, pulsed dexamethasone, and pulsed intravenous methylprednisolone. J Neurol 2018; 265 (9): 2052-9.

29. Ülkü Türk Börü, Hülya Erdoğan, Recep Alp, Mustafa Taşdemir, Serhan Yıldırım, et al. Treatment of chronic inflammatory demyelinating polyneuropathy with high dose intravenous methylprednisolone monthly for five years: 10-year follow up Clin Neurol Neurosurg 2014; $118: 89-93$.

30. Allen JA, Lewis RA. CIDP diagnostic pitfalls and perception of treatment benefit. Neurology 2015; 85 (6): 498-504. 